\title{
A stringy test of the fate of the conifold
}

\section{Citation}

Vafa, Cumrun. 1995. "A Stringy Test of the Fate of the Conifold." Nuclear Physics B 447 (2-3):

252-60. https://doi.org/10.1016/0550-3213(95)00279-2.

\section{Permanent link}

http://nrs.harvard.edu/urn-3:HUL.InstRepos:41385056

\section{Terms of Use}

This article was downloaded from Harvard University's DASH repository, and is made available under the terms and conditions applicable to Other Posted Material, as set forth at http:// nrs.harvard.edu/urn-3:HUL.InstRepos:dash.current.terms-of-use\#LAA

\section{Share Your Story}

The Harvard community has made this article openly available.

Please share how this access benefits you. Submit a story.

Accessibility 
HUTP-95/A014

\title{
A Stringy Test of the Fate of the Conifold
}

\author{
Cumrun Vafa \\ Lyman Laboratory of Physics \\ Harvard University \\ Cambridge, MA 02138, USA
}

\begin{abstract}
By studying string loop corrections to superpotential of type II strings compactified on Calabi-Yau threefolds we find a quantum stringy test and a confirmation of a recent proposal of Strominger on the fate of the conifold singularity. We also propose a connection between the spectrum of Bogomolnyi saturated solitons and one-loop string partition function of $N=2$ topological strings.
\end{abstract}


Through some recent exciting work in string theory it has become clear that nonperturbative aspects of string theory are within reach, at least for certain string compactifications. In particular a clear picture is emerging for qualitative nonperturbative aspects of string theory for type II string compactifications on tori [1] [2], on $K 3$ manifold [1] [2] [3] and on Calabi-Yau threefolds [4] [5]. In this paper we present some evidence based on string loop corrections in favor of the beautiful picture advocated in [4] for non-perturbative aspects of type II strings on Calabi-Yau manifolds. An argument in favor of the scenario suggested in [4] is the exciting discovery [5] that it allows smooth transitions among Calabi-Yau manifolds, thus realizing a suggestion made in [6] [7] [9]. It is amusing that string loop corrections can be used to test some of these non-perturbative aspects of string theory. This is in accord with a type IIA string one loop computation [10] which further supports the conjecture [1] [1] that type IIA compactified on $K 3$ is dual to toroidal compactification of heterotic string down to 6 dimensions.

Let us recall the basic picture advocated in [4] for the explanation of the conifold singularity in Calabi-Yau compactifications. Type IIB strings compactified on a Calabi-Yau threefold leads to a theory in $4 \mathrm{~d}$ with $N=2$ supersymmetryt. The massless string states apart from the gravity multiplet include some $U(1)$ vector multiplets and some hypermultiplets. The number of vector multiplets is equal to $h^{2,1}$ of the Calabi-Yau manifold $M$ and the number of hypermultiplets is equal to $h^{1,1}+1$. Since we have $N=2$ supersymmetry in 4 dimensions we can have a central charge in the supersymmetry algebra. This could lead to BPS states which saturate the Bogomolnyi bound and are thus stable. Such objects would have electric or magnetic charge under the $U(1)$ gauge fields of the vector multiplet. However there are no such states among the fundamental string excitations as they are all neutral under the Ramond-Ramond $U(1)$ gauge fields. It was suggested in [4] that by wrapping certain threebrane solutions [12] on three-cycles of Calabi-Yau one could construct extreme black hole solutions in four dimensions which would carry $U(1)$ charges. Assuming charge quantization the allowed electric and magnetic charges of such states would be in one to one correspondence with $H_{3}(M, \mathbf{Z})$. Let $C_{i}$ be an integral three-cycle of $M$. Let $\omega$ denote the holomorphic three form on $M$. Then the central charge of the supersymmetry algebra $W_{i}$ (up to normalization) for this black hole is given by

$$
W_{i}=\int_{C_{i}} \omega
$$

1 It suffices to consider type IIB, because type IIA is equivalent to the same theory on the mirror Calabi-Yau. 
and the BPS mass is given by

$$
m_{i}^{2}=\frac{\int_{C_{i}} \omega \cdot \int_{C_{i}} \bar{\omega}}{\int_{M} \omega \wedge \bar{\omega}}
$$

(for a recent discussion on this see [13]). The question of whether all such classical solutions, and with what multiplicities, lead to quantum states is not clear. In fact for the resolution of the conifold singularity it was argued in [4 that not all of such black hole states exist as fundamental fields in the quantum theory. Consider a conifold point $p$ of the CalabiYau moduli space. This is a point where by definition there exists a 3 -cycle $C_{p}$ for which $W_{p}=\int_{C_{p}} \omega \rightarrow 0$ as we approach the conifold point (assuming a normalization of $\omega$ so that $\left.\int \omega \wedge \bar{\omega}>0\right)$. A conifold point is a finite distance away from the interior of the moduli space, and the moduli space has a curvature singularity at that point. This would cause problem for the predictive power of string theory. This problem is more acute because one can show that the moduli space metric is perturbatively uncorrected. As has been elaborated in [14] the special geometry metric on the moduli space of Calabi-Yau manifolds can be understood as an effective field theory for the massless modes at the string tree level, where one integrates out massive Kaluza-Klein states of the Calabi-Yau. This in particular means that the origin of this tree level singularity in string theory can be understood by the interaction of the moduli fields with massive string states that are becoming massless at the conifold point. This however is not the end of the story in the full quantum theory of strings. Even though we can understand the classical origin of the singularity from the viewpoint of nearly massless string states, such states are not stable even at the tree level and thus could hardly make sense in the full quantum theory.

We thus have two possibilities: Either we can assume these singularities are not there when we consider non-perturbative string effects, or their origin in the full theory can be understood by some other nearly massless state. The latter was the viewpoint advocated in 团. This is somewhat similar to what happens for $N=1$ supersymmetric Yang-Mills in $4 \mathrm{~d}$ with $G=S U\left(N_{c}\right)$ and with $N_{f}=N_{c}+1$ where the moduli space is classically singular and is uncorrected by quantum effects, but the explanation of the singularity in the full theory is different [15], where it is explained in terms of massless Baryons and mesons.

It was suggested in [4] that the metric on the moduli space is not corrected even non-perturbatively and furthermore near a conifold point $p$ the stable nearly massless field exists which is a black hole state corresponding to the vanishing cycle $C_{p}$. Such black hole states can be constructed by wrapping the threebrane solution of [12] around a three-cycle of the Calabi-Yau manifold. For this to work it was crucial to assume that there are no 
stable black holes corresponding to the cycles $n C_{p}$ for $|n|>1$. The proposed black hole is an $N=2$ hypermultiplet. Note that if we start from the interior of the moduli space and approach the conifold point $p$ the cycle that becomes a vanishing cycle will depend on the path that we take to $p$. It is natural then to consider the totality of such vanishing cycles at each point on the moduli space as they would be a natural candidate for the space of stable black holes. The totality of such vanishing cycles can be obtained by considering the action of the monodromy group of the moduli on a basis for the vanishing three-cycles. The monodromy group is obtained by deleting codimension one singularities of the moduli space (including orbifold points) and considering the transformation of three-cycles around such singularities. This will be a subgroup $H \subset S p\left(h^{2,1}+1, \mathbf{Z}\right)$. Thus if $C_{p}$ is any one of the vanishing cycles (and a primitive three-cycle in the homology of $M$ ), we consider the space

$$
\mathcal{V}=\left\{H \cdot C_{p}\right\}
$$

This is a candidate for at least a subspace of stable black holes. The cycles in $\mathcal{V}$ would all have to correspond to stable black hole states were it not for the fact that BPS states may appear or disappear when the Bogomolnyi charges of two cycles are in the same direction in the complex plane. In particular suppose $C_{i}$ and $C_{j}$ correspond to occupied black hole states, with Bogomolnyi charges given by $W_{i}$ and $W_{j}$. Then if

$$
W_{i}=\alpha W_{j} \quad \alpha>0
$$

where $\alpha$ is a positive real number, then a black hole state corresponding to the cycle $C_{i}+C_{j}$ can appear or disappear. This does happen in massive $N=2$ models in 2 dimensions which was discovered and investigated in detail in [16] [17]. In that case the BPS states were related to the intersection numbers of the vanishing cycles of the Landau-Ginzburg singularity and the monodromy of the vanishing cycle leads to a jump in the intersection numbers and thus the number of BPS solitons. It is tempting to speculate here as well that the intersection numbers of the vanishing cycles is related to the soliton number jumping. Note that since the cycles $C_{i}$ are in dimension 3, their intersection makes sense, just as was the case considered in [16]. Similar phenomenon of jumping of BPS solitons were also postulated in [18 in order to obtain a consistent exact picture of 4 dimensional $N=2$ theories. Given that here we are also dealing with $N=2$ theories in 4 dimensions it is likely that we will also have the jumping phenomenon. Note that the jumping phenomenon is inherently associated with having a complex Bogomolnyi charge as is the case with $N=2$ 
supersymmetry. In such cases if one wishes to move in the moduli of the theory in certain cases one cannot avoid passing through configurations with collinear Bogomolnyi charges along a path joining two given points on the moduli. This is the reason for the possibility of having jumping. If we have $N>2$ we do not expect such jumping phenomenon because the Bogomolnyi charge lives in a bigger space, and in going from an initial point in the moduli to a final point, we can avoid passing through collinear Bogomolnyi charges. Since whether we have jumping or not should depend only on the end points on the moduli space this means we do not have any jumping if $N>2$.

The tree level singularity near the conifold can be explained by the existence of a nearly massless black hole state in the full theory. Can we test this idea further using perturbative string amplitudes? If we encounter quantum string amplitudes which diverge as we approach the conifold point, then there should be an alternative explanation of the singularity from the viewpoint of the existence of massless black holes. The main difficulty, apart from the practical question of computation is that any computation we do may have correction at all loops and even non-perturbatively. We must thus consider objects which are not corrected to all orders in perturbation theory and also non-perturbatively. In fact it has been argued that superpotential terms considered upon compactification of type II strings on Calabi-Yau manifolds are of this type and moreover can be computed in string theory [14] 19] by relating them to $N=2$ topological string amplitudes on the Calabi-Yau manifold. In particular the genus $g$ topological amplitude $F_{g}$ computes, as a function of the moduli, a correction to the lagrangian. These corrections involve superpotential terms for the vector multiplet and can be written conveniently in the superspace by (with a similar formula for the hypermultiplets [19])

$$
\delta S=\int d^{4} \theta F_{g} W^{2 g} \sim \int F_{g} R^{2} T^{2 g-2}+\ldots
$$

where $W$ is the $N=2$ superfield associated to graviphoton field strength, $R$ is the Riemann tensor, and $T$ is the graviphoton field strength. The contractions in the above terms are fixed by supersymmetry. The status of the non-perturbative correction to these terms is on the same footing as the special geometry. So it is reasonable to postulate that they do not get corrected even non-perturbatively and so $F_{g}$ computes the full answer for the correction of $W^{2 g}$ to the theory.

The topological amplitudes $F_{g}$ has been computed for some Calabi-Yau manifolds for small $g$ [14] 20]. Moreover the structure of the singularity near the conifold point was 
determined for all $g$ in [14]. $F_{1}$ has in addition been studied for many more examples including some with 2 parameter moduli space [21]. Since we are now taking $F_{g}$ to correspond to certain exact computations in the full theory we should be able to understand their singularity from the viewpoint of nearly massless black holes. This is what we will presently do.

In all the examples considered in [14][20] 21] there was a single conifold point $p$ (and codimension one in the 2 parameter family studied in [21]). Let $z$ denote the period of the vanishing cycle as we approach $p$. In particular $z(p)=0$. The singularity that was encountered is of the form

$$
\begin{gathered}
F_{1}=\frac{-1}{12} \log z \bar{z}+\ldots \\
F_{g}=\frac{\text { const. }}{z^{2 g-2}}+\ldots \quad g>1
\end{gathered}
$$

The precise coefficient of singularity for $F_{1}, \frac{-1}{12}$, was computed by fixing behaviour of $F_{1}$ at other boundaries of moduli space where there was a better understanding of its behaviour. It was thus quite mysterious that in all the four examples considered in [20] and all the other examples including the 2 parameter family ones studied in [21], the coefficient of the conifold singularity came out to by $-1 / 12$ (there is an extra factor of $1 / 2$ relative to that in [20]; as explained in [14] this comes from the $Z_{2}$ symmetry of the torus). Let us first consider the genus 1 term. In this case (2) shows that we are computing a term of the form $R^{2}$. In order to fix the absolute normalizations of string one loop computation it turns out to be convenient to consider a part of the interaction given by the index contraction leading to the Euler characteristic density. The rest will be determined by $N=2$ supersymmetry. The idea is to consider further compactification on a four manifold- in this case in the computation of the string one-loop partition function the term corresponding to the Eulercharacteristic would come from the sector where both left- and right-movers have the same spin structure. If the spin structure is odd, it clearly gives the Euler characteristic $\chi$. If we consider the sum of the 9 spin structures where they are even on both left- and rightmovers, by supersymmetry one can show that we also get $\chi$. Now, there is an overall factor of $1 / 4$ coming from GSO projection factor. So putting all this together we find that the precise string one loop normalization is such that it gives

$$
\delta_{1} S=\frac{1 / 2}{128 \pi^{2}} \int F_{1} \epsilon^{a b i j} \epsilon^{c d k l} R_{a b c d} R_{i j k l}=\frac{F_{1} \cdot \chi}{2}
$$


We are thus predicting a correction to the effective action which near the conifold point is of the form

$$
\delta_{1} S=\frac{-\chi}{24} \log z \bar{z}=\frac{-\chi}{24} \log m^{2}
$$

Where would this come from in the effective theory where we have a nearly massless black hole with mass $m$ ? Counting the powers of derivatives we see that it should come from a one loop computation in the effective theory (which is also responsible for giving the tree level string singularity as suggested in [4]). Indeed it is easy to see that such a term is generated at one loop. At one loop we would have to compute

$$
\frac{-1}{2} \mathrm{~S} \operatorname{Tr} \log \left(-D^{2}+m^{2}\right)=\frac{-1}{2} \int_{\epsilon}^{\infty} \frac{d s}{s} \operatorname{Tr}(-1)^{F} \exp \left[-s\left(-D^{2}+m^{2}\right)\right]
$$

where $D^{2}$ is the Laplacian acting on the fields in the multiplet and $(-1)^{F}$ is \pm 1 depending on whether we are dealing with a boson or a fermion in the multiplet. The term in this expansion proportional to $\log m^{2}$ comes from the order $s^{0}$ expansion of the heat kernel $\operatorname{Tr}(-1)^{F} \exp \left[-s\left(-D^{2}\right)\right]$. The order $s^{0}$ term can be expanded in the metric, and by dimension counting it includes terms with four derivatives. Suppose we have $N$ hypermultiplets. Recall that a hypermultiplet has 2 complex scalars and 2 Weyl fermions. Thus we find a one loop term of the form

$$
\delta S=\frac{-N \log m^{2}}{2}\left[4 b_{4}(0,0)-b_{4}\left(\frac{1}{2}, 0\right)-b_{4}\left(0, \frac{1}{2}\right)\right]
$$

where $b_{4}(n, m)$ denotes the coefficient of heat kernel expansion for a particle of spin $(n, m)$ under $S O(4)$. These have been computed in the literature [22]. Again by $N=2$ supersymmetry it suffices to consider the term proportional to Euler characteristic. It turns out that

$$
4 b_{4}(0,0)-b_{4}\left(\frac{1}{2}, 0\right)-b_{4}\left(0, \frac{1}{2}\right)=\frac{\chi}{12}+\ldots
$$

We thus find for $N$ hypermultiplets of mass $m$ a term in the effective action of the form

$$
\delta S=\frac{-N \chi \log m^{2}}{24}
$$

Comparing this with the exact string theory computation (4) we get agreement if $N=1$ as was required for the consistency of the picture advocated in [四. This also 'explains' the mysterious factor of $-1 / 12$ appearing in all the examples studied in [20] [21].

Note that the special geometry of moduli of Calabi-Yau manifold has a bearing on the coupling of $F^{2}$ as a function of moduli, whereas the one loop string computation 
discussed above has a bearing on the coupling of $R^{2}$. In particular the tree level amplitude is sensitive only to charged states, whereas the one loop term, being a gravity effect, takes into account all states. In particular in finding an agreement between the one loop and tree level counting of massless states we are finding evidence that there are no additional neutral fields which are becoming massless at the conifold point 2 . It is amusing that also for string-string duality in six dimensions in order to reproduce the sigma-model anomaly of heterotic string in the type IIA string, it was found [10 that the tree level gives the $F^{2}$ part and the one loop gives the $R^{2}$ part.

In the effective theory near the conifold not only the nearly massless hypermultiplet generates a one loop term proportional to $\chi$, but all hypermultiplets do. It is thus tempting to go further and identify $F_{1}$ with a partition function for all BPS states:

$$
F_{1}=\frac{-1}{12} \sum_{B P S \text { states }} \log m_{i}^{2}
$$

where the above sum on the right hand side may need to be regularized and where $m_{i}^{2}$ is given by the formula (1). This may not be precisely right: There is a jumping phenomenon and so the BPS states do not necessarily form a modular invariant subspace, however $F_{1}$ is modular invariant. Moreover in the effective theory describing the vicinity of the conifold there may be other terms in the action proportional to $\chi$. Another check on (6) is to study the anomaly equation it satisfies [20]. By considering moduli derivatives $\partial \bar{\partial} F_{1}$, if (6) were to hold, we see using (1) that it would be proportional to $\partial \bar{\partial} K$ where $\exp (-K)=\int_{M} \omega \wedge \bar{\omega}$ which according to [20] is only a piece of the anomaly satisfied by $F_{1}$. In fact $F_{1}$ is given by

$$
F_{1}=\log \left[\exp \left[\left(\frac{3}{2}+\frac{h^{2,1}}{2}+\frac{\chi}{24}\right) K\right] \operatorname{det} G^{-1 / 2}|f|^{2}\right]
$$

where $f$ is a holomorphic function of moduli and $G=\partial \bar{\partial} K$ is the metric on the moduli space. The extra non-holomorphicity comes from non-holomorphicity of $G$. But we also have to recall that in considering BPS states in (6) we have to take into account the massless fields corresponding to the moduli. The factor of $\log \operatorname{det} G^{-1 / 2}$ is exactly right to come from the normalization of the path integral for the massless moduli field. At any rate, even if $F_{1}$ is not precisely the partition function of BPS states, I believe (6) should be very

2 It could happen, however, that we have extra massless hypermultiplets and vector multiplets, equal in number, since the contribution of vector multiplets turns out to be opposite in sign to that of hypermultiplet in the coefficient of $R^{2}$. 
closely related to it, and should be an important ingredient in fixing all the BPS states. This is analogous to ideas suggested in [23] in constructing automorphic forms on moduli space of Calabi-Yau and relating it to sums over specific three-cycles. To motivate it even further let us recall that $F_{1}$ computes a specific combination of Ray-Singer torsions on the Calabi-Yau threefold [14], i.e. a combination of logarithm of the determinant of Laplacian acting on forms of the Calabi-Yau manifold. Let us consider an analogy: Suppose we consider a one dimensional Calabi-Yau, i.e. a torus. In that case the analog of the threecycles on the threefold are the one-cycles on the torus. Then the analog of candidates for BPS states would be one-cycles and the analog of the formula (6) is

$$
F_{1}=-\log \left(\operatorname{det} D^{2}\right)=\sum_{n, m} \log \frac{|n+m \tau|^{2}}{\tau_{2}}
$$

which is a correct relation. Thus in the threefold we would be suggesting a relation between the particular combination of Ray-Singer torsions appearing in [14] and a subspace of threecycles in the Calabi-Yau manifold corresponding to BPS solitons. It should be interesting to develop this further. In particular it should be interesting to see what happens if we restrict the sum in (6) to the space of vanishing cycles $\mathcal{V}$ discussed before. Another interesting point to study would be the behavior of $F_{1}$ for boundaries of moduli space which are infinitely far away (analogous to infinite volume) and study what it teaches us about the full spectrum of the theory in that limit.

It is easy to see by power counting that in order to obtain the higher genus superpotential terms computed in string theory, we need higher loop corrections in the effective theory as well. In such a scenario near the conifold point the powers of $m^{2}$ in the denominator in (3) would come from infrared divergencies associated with a nearly massless black hole. Power counting suggests that such a singularity could appear in a $g$-th loop correction in the effective theory; for example having a loop of nearly massless black holes with internal graviton exchanges will give the correct power counting to reproduce the effective action computed in string theory.

So far we have talked about compactifications of type II strings on Calabi-Yau manifolds down to 4 dimensions. If we instead consider type II strings compactified on $K 3$ to 6 dimensions, there are superpotential terms that are again captured by a topological string theory: The $N=4$ topological string [24] which is equivalent to $N=2$ strings. Just as

3 Summing over primitive one-cycles will give the same result. 
the above superpotential terms generated upon compactification on Calabi-Yau is relevant in studying certain non-perturbative aspects of string theory, it is natural to expect that the $N=2$ string is important in a better understanding of heterotic-type IIA duality in six dimensions. This question is currently under investigation [25].

I would like to thank N. Berkovits, M. Bershadsky, S. Kachru, H. Ooguri, M.H. Sarmadi, A. Strominger, E. Witten and S.-T. Yau for valuable discussions.

This research was supported in part by the NSF grant PHY-92-18167. 


\section{References}

[1] C.M. Hull and P.K. Townsend, "Unity of Superstring Dualities", QMW-94-30, $\mathrm{R} / 94 / 33$.

[2] E. Witten, "String Theory Dynamics in Various Dimensions", hep-th/9503124.

[3] A. Sen, "String-String Duality Conjecture in Six Dimensions and Charged Solitonic Strings", hep-th/9504027;

J. Harvey and A. Strominger, "The Heterotic String is a Soliton", hep-th/9504047

[4] A. Strominger, "Massless Black Holes and Conifolds in String Theory", hep-th/950490

[5] B. Greene, D. Morrison and A. Strominger, "Black Hole Condensation and the Unification of String Vacua", hep-th/9504145.

[6] P. Candelas, A.M. Dale, C.A. Lütken, and R. Schimmrigk, "Complete Intersection Calabi-Yau Manifolds", Nucl. Phys. B298 (1988) 493-525.

[7] P.S. Green and T. Hübsch, "Possible Phase Transitions Among Calabi-Yau Compactifications", Phys. Rev. Lett. 61 (1988) 1163-1166; "Connecting Moduli Spaces of Calabi-Yau Threefolds", Comm. Math. Phys. 119 (1988) 431-441.

[8] P. Candelas, P.S. Green, and T. Hübsch, "Finite Distance Between Distinct CalabiYau Manifolds", Phys. Rev. Lett. 62 (1989) 1956-1959; "Rolling Among Calabi-Yau Vacua", Nucl. Phys. B330 (1990) 49-102.

[9] P. Candelas and X.C. de la Ossa, "Comments on Conifolds", Nucl. Phys. B342 (1990) 246-268.

[10] C. Vafa and E. Witten, in preparation.

[11] C. Vafa, unpublished.

[12] G. Horowitz and A. Strominger, "Black Strings and p-branes," Nucl. Phys. B360 (1991) 197.

[13] A. Ceresole, R. D'Auria, S. Ferrara and A. Van Proeyen, "Duality Transformations in Supersymmetric Yang-Mills Theories coupled to Supergravity",hep-th/9502072.

[14] M. Bershadsky, S. Cecotti, H. Ooguri and C. Vafa, "Kodaira-Spencer Theory of Gravity and Exact Results for Quantum String Amplitudes", Comm. Math. Phys. 165 (1994) 311.

[15] N. Seiberg, "Exact Results on the Space of Vacua of Four Dimensional SUSY Gauge Theories", hep-th 9402044.

[16] S. Cecotti and C. Vafa, "On Classification of N=2 Supersymmetric Theories", Comm. Math. Phys. 158 (1993) 569.

[17] S. Cecotti, P. Fendley, K. Intriligator and C. Vafa, " A New Supersymmetric Index", Nucl. Phys. B386 (1992) 405.

[18] N. Seiberg and E. Witten, "Electromagnetic Duality, Monopole Condensation and Confinement in $N=2$ Supersymmetric Yang-Mills Theory", Nucl. Phys. B426 (1994) $19-52$. 
[19] I. Antoniadis, E. Gava, K.S. Narain and T.R. Taylor, "Topological Amplitudes in String Theory", Nucl. Phys. B413 (1994) 162.

[20] M. Bershadsky, S. Cecotti, H. Ooguri and C. Vafa, "Holomorphic Anomalies in Topological Field Theories", Nucl. Phys. B405 (1993) 279.

[21] S. Hosono, A. Klemm, S. Theisen and S.-T. Yau, "Mirror Symmetry, Mirror Map and Applications to Complete Intersection Calabi-Yau Spaces", Nucl. Phys. B433 (1995) 501 ;

P. Candelas, A. Font, S. Katz and D.R. Morrison, "Mirror Symmetry for Two Parameter Models", Nucl. Phys. B429 (1994) 626.

[22] B.S. DeWitt, "Dynamical Theory of Groups and Fields", (Gordon and Breach, New York 1965);

P.B. Gilkey, J. Diff. Geom. 10 (1975) 601;

S.M. Christensen and M.J.Duff, Nucl. Phys. B154 (1979) 340.

[23] S. Ferrara, C. Kounnas, D. Lust and F. Zwirner, "Duality-Invariant Paritition Functions and Automorphic Superpotentials for $(2,2)$ String Compactifications", Nucl. Phys. B365 (1991) 431.

[24] N. Berkovits and C. Vafa, "N=4 Topological Strings", HUTP-94/A018, KCL-TH-9412.

[25] N. Berkovits, H. Ooguri and C. Vafa, Work in progress. 